\title{
Maximum thickness of a two-dimensional trapped Bose system
}

\author{
Sang-Hoon Kim \\ Division of Liberal Arts, Mokpo National Maritime University, Mokpo 530-729, Korea
}

\begin{abstract}
The trapped Bose system can be regarded as two-dimensional if the thermal fluctuation energy is less than the lowest energy in the perpendicular direction. Under this assumption, we derive an expression for the maximum thickness of an effective two-dimensional trapped Bose system.
\end{abstract}

PACS numbers: 05.30.Jp, 03.75.F, 68.65.+g

It has been known that the Bose-Einstein Condensation (BEC) cannot occur in either two-dimensional (2D) or one-dimensional (1D) uniform Bose gas at a finite temperature because thermal fluctuations destabilize the condensate [1]. However, when a spatially varying potential which breaks the translational invariance exists, the BEC may occur in low dimensional inhomogeneous systems. In the presence of harmonic trapping, the effect of thermal fluctuations are strongly quenched due to the different behavior exhibited by the density of states.

In three-dimensional (3D) traps, the experimental results for the BEC have been obtained assuming that the thermal fluctuation energy $k_{B} T$ is much larger than all the oscillator energies $\left(\hbar \omega_{x}, \hbar \omega_{y}, \hbar \omega_{z}\right)$. In order to achieve a $2 \mathrm{D}$ BEC in the trap, it is necessary to choose the frequency $\omega_{z}$ large enough to satisfy the condition $\hbar \omega \ll k_{B} T_{2 D} \ll \hbar \omega_{z}$, where $\omega=\sqrt{\omega_{x} \omega_{y}}$ and $T_{2 D}$ is the $2 \mathrm{D}$ transition temperature. This is a rather difficult condition to satisfy in the trap design, and leads the realization of a 2D system in another way.

Recently, Safonov et al. [2] have reported an observation of a quasi-2D BEC in liquid hydrogen layers. They successfully confined hydrogen atoms on a liquid ${ }^{4} \mathrm{He}$ surface which corresponds to a potential well of $20 \mu \mathrm{m}$ width. Also, Gauck et al. 3 claimed that they achieved another quasi-2D system of argon atoms confined in a planer matter waveguide in the close vicinity of $\mu \mathrm{m}$. However, the question that up to what thickness we can regard the system as $2 \mathrm{D}$ is remained. The mathematical concept of $2 \mathrm{D}$ which neglects one (z-direction) degree of freedom and allows particles to move only in surface (x-y plane) is not physically acceptable due to the uncertainty principle. In this communication, we suggest a criterion for the BEC to exhibit 2D behavior in 3D space, and obtain a maximum thickness of the 2D trapped Bose system.

In the 2D experimental setup, the z-directional thickness corresponds to ideal rigid walls. For the infinite potential well of $-d / 2 \leq z \leq d / 2$, the lowest energy is given by $E_{g}=\hbar^{2} \pi^{2} / 2 m d^{2}$. The system can be regarded as $2 \mathrm{D}$ as long as the thermal fluctuation energy is less than the z-directional lowest energy. That is,

$$
k_{B} T_{2 D}<\frac{\hbar^{2} \pi^{2}}{2 m d^{2}} .
$$

The transition temperature of the BEC is not precisely known except for the ideal Bose gas in the harmonic trap. Although the systems used in the experiment were not ideal, the measured transition temperatures in 3D were found to be very close to the ideal gas value. A similar situation is expected in 2D. The transition temperature of the 2D BEC for the ideal Bose gas system in the harmonic trap is given by [4]

$$
k_{B} T_{2 D}=\hbar \omega\left(\frac{N}{\zeta(2)}\right)^{1 / 2}
$$

where $N$ is the number of atoms in the trap and $\zeta(x)$ is the Riemann-Zeta function.

Substituting Eq. (2) into Eq. (1), we obtain an effective $2 \mathrm{D}$ thickness of

$$
\frac{d}{a_{h o}}<\frac{\pi}{\sqrt{2}}\left(\frac{\zeta(2)}{N}\right)^{1 / 4},
$$

or

$$
(\text { Thickness of } 2 \mathrm{D})<\frac{2.516}{N^{1 / 4}} a_{h o} \text {, }
$$

where $a_{h o}$ is the harmonic oscillator length given as $a_{h o}=\sqrt{\hbar / m \omega}$. We note that a typical value for $a_{h o}$ is of the order of $\mu \mathrm{m}$ for alkali Bose atoms and much larger for hydrogen atoms.

In conclusion, we have obtained a maximum value of the effective thickness of a 2D trapped Bose system in which to observe the BEC. The maximum radius of a $1 \mathrm{D}$ trapped Bose system could be obtained in a similar way.

We thank Professors C.K. Kim, K. Nahm, and M. Chung for useful discussions.

[1] P.C. Hohenberg, Phys. Rev. 158, 383 (1967).

[2] A.I. Safonov, S.A. Vasilyev, I.S. Yasnikov, I.I. Lukashevich, and S. Jaakola, Phys. Rev. Lett. 81, 4545 (1998).

[3] H. Gauck, M. Hartl, D. Schneble, H. Schnitzler, T. Pfau, and J. Mlynek, Phys. Rev. Lett. 81, 5298 (1998).

[4] W.J. Mullin, J. Low Temp. Phys. 106, 615 (1997). 